\title{
How Does the Camera Assistant Decide the Zooming Ratio of Laparoscopic Images? Analysis and Implementation
}

\author{
Atsushi Nishikawa ${ }^{1}$, Hiroaki Nakagoe ${ }^{1}$, Kazuhiro Taniguchi ${ }^{1}$, Yasuo Yamada ${ }^{1}$, \\ Mitsugu Sekimoto ${ }^{2}$, Shuji Takiguchi ${ }^{2}$, Morito Monden ${ }^{2}$, and Fumio Miyazaki ${ }^{1}$ \\ ${ }^{1}$ Department of Mechanical Science and Bioengineering, \\ Graduate School of Engineering Science, Osaka University, Japan \\ ${ }^{2}$ Department of Gastroenterological Surgery, \\ Graduate School of Medicine, Osaka University, Japan
}

\begin{abstract}
An important factor for defining a good image during laparoscopic surgery is the zooming ratio, which corresponds to the depth of insertion of the laparoscope along its longitudinal axis. However, it is not clear how surgeons (camera assistants) decide the zooming ratio of laparoscopic images during surgery. Conventional automatic camera positioning systems define the zooming ratio "uniformly" based on simple heuristics. However, because the most adequate zooming ratio varies widely during surgery, these conventional systems may not offer the specific view that the surgeon wants. Therefore, we first investigated how the camera assistant decides the zooming ratio of laparoscopic images by fully analyzing the positional relationship between the laparoscope and the surgical instrument during laparoscopic surgery. Then, we extracted the zooming behavior and implemented it in the robotic laparoscope positioner that we previously developed. As a result, the zooming behavior of our robotic system became very similar to that of the human camera assistant. It was found that the proposed zooming motion of our robotic system may be suitable for fast and compact operations during surgery.
\end{abstract}

\section{Introduction}

In current laparoscopic surgery, the visual field of the operating surgeon usually depends on the camera assistant responsible for guiding the laparoscope. The assistant holds the laparoscope for the surgeon and positions the scope according to the surgeon's instructions. This procedure can be frustrating and inefficient for the surgeon, because the assistant often erroneously interprets and executes the commands. Also, the views can be suboptimal and unstable because the scope is sometimes aimed incorrectly and vibrates due to the assistant's hand tremors. To solve these problems, several robotic laparoscope positioning systems have been developed in the last fifteen years. Although these camera positioners may provide more precise positioning of the laparoscope as well as stable images, they usually are controlled by the operating surgeon himself/herself using a human-machine interface such as an instrument-mounted joystick, foot pedal, 
voice controller, or head/face motion-activated system. This is an additional task that distracts the surgeon's attention from the main region of interest and may result in frustration and longer surgery time.

To free the surgeon from the task of controlling the view and to automatically offer an optimal and stable view during laparoscopic surgery, several automatic camera positioning systems have been devised 1123456. These systems visually extract the shape and/or position of the surgical instrument from the laparoscopic images in real time, and automatically manipulate the laparoscope to always center the tip of the instrument in the displayed image. Such systems are based on the simple idea that the projected position of the distal end of the surgical tool corresponds to the surgeon's region of interest in a laparoscopic image. Besides centering on the most interesting area, there is an additional and important factor that defines a good image of the surgical scene - zooming ratio - that corresponds to the depth of insertion of the laparoscope along its longitudinal axis. The pioneering studies of fully automatic camera positioning systems defined the zooming ratio as a "uniform" function of the estimated distance between the tip of the tool and the laparoscope [1] or the area ratio between the visible tool and the whole image [2]. Although these approaches may completely remove the surgeon's camera control burden, they may not provide the specific view that the surgeon wants, because the most appropriate zooming ratio varies widely during surgery. The best zooming ratio depends on both the surgical procedure/phase and the habits/preferences of the operating surgeon. For this reason, most of the instrument tracking systems recently developed [3,4,5,6] have abandoned the idea of systematic control of zooming parameters; instead, the surgeon is required to define the parameters preoperatively or adjust them intraoperatively through conventional human-machine interfaces, which again means an extra control burden for the surgeon.

To overcome this problem, we first investigated how the camera assistant decides the zooming ratio of laparoscopic images by fully analyzing the positional relationship between the laparoscope and the surgical instrument during laparoscopic surgery. Then, we extracted the zooming behavior and implemented it in a robotic laparoscope positioner that we have developed. As a result, the zooming behavior of our robotic system became very similar to that of the human camera assistant. It was found that the proposed zooming motion in the robotic system can be suitable for fast and compact operations during laparoscopic surgery.

\section{Analysis of Zooming Behavior of Camera Assistants}

\subsection{Measurement System Overview}

In order to analyze the "zooming" behavior of the human camera assistant who maneuvers the laparoscope during surgery, a number of tests were performed. As shown in Figure 1. one test was a simple laparoscopic cholecystectomy simulation; that is, a pig liver with a gallbladder was placed in the training box and the gallbladder was removed by an operating surgeon with the use of a laparoscope controlled by another surgeon (camera assistant). A 3D tracking system (Polaris 

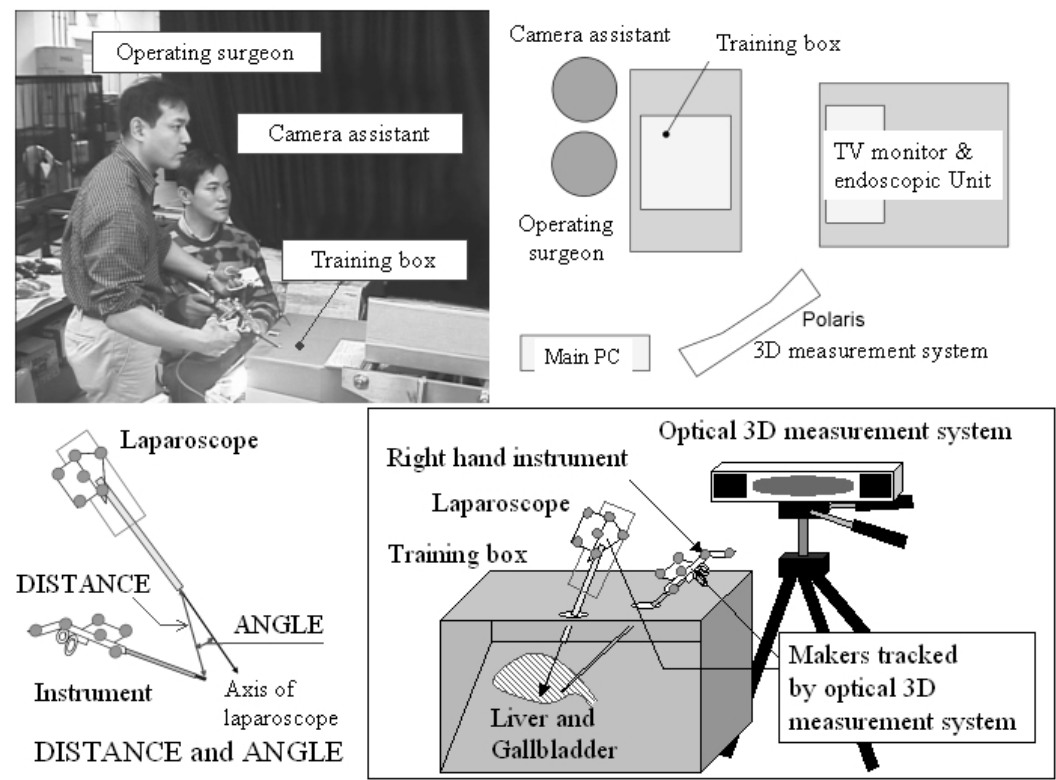

Fig. 1. Measurement system

Accedo, NDI Corporation) was used. This system requires software predefinition of the physical structure of a marker composed of at least three reference points. Each reference point is a small sphere covered with highly reflective material. The tracking system is capable of determining the 3D position/pose of a marker at a frequency of $20 \mathrm{~Hz}$, as long as a sufficient number of reference points of the marker are visible. In order to gather the positional relationship between the surgical instrument and the laparoscope during surgery, two different markers were designed: one was attached to the top of the right-hand instrument and other to the laparoscope. Both markers were much less likely to be lost by the tracking system because each was composed of six reference points. With six points, at least three points remained visible to determine the marker's location in the world coordinate system [7].

\subsection{Definition of Distance and Angle}

We focused on the following two parameters: DISTANCE and ANGLE (see Figure 1). Let DISTANCE be the distance between the tip of the laparoscope and the tip of the right-hand instrument. Also, let ANGLE be the angle between the direction of the longitudinal axis of the laparoscope and the direction from the tip of the scope to the tip of the right-hand instrument. Both DISTANCE and ANGLE can be calculated in real time from the output of the tracking system and known geometric information about the instrument and the laparoscope. 


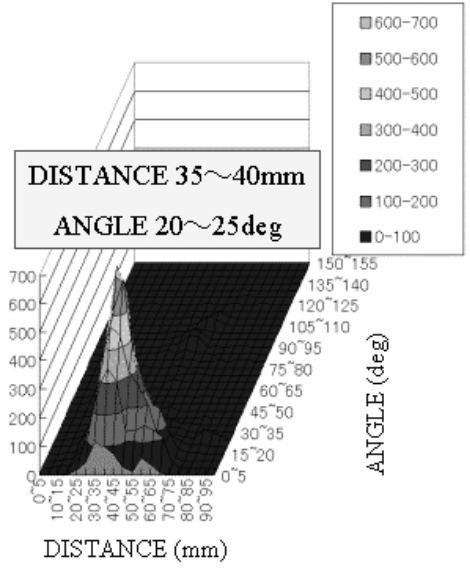

Dr. A \& Dr. B

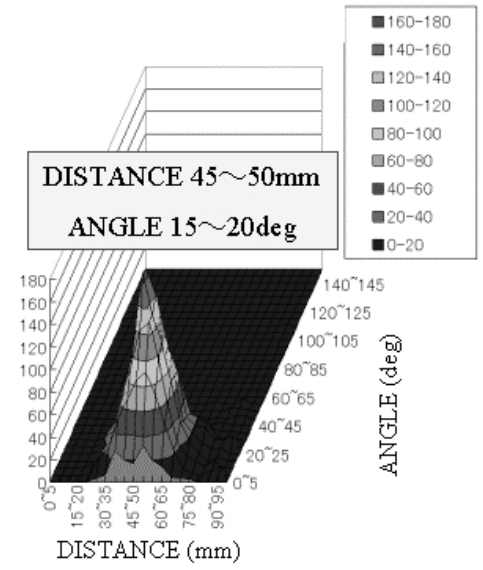

Dr. C \& Dr. D

Fig. 2. DISTANCE-ANGLE distribution histograms

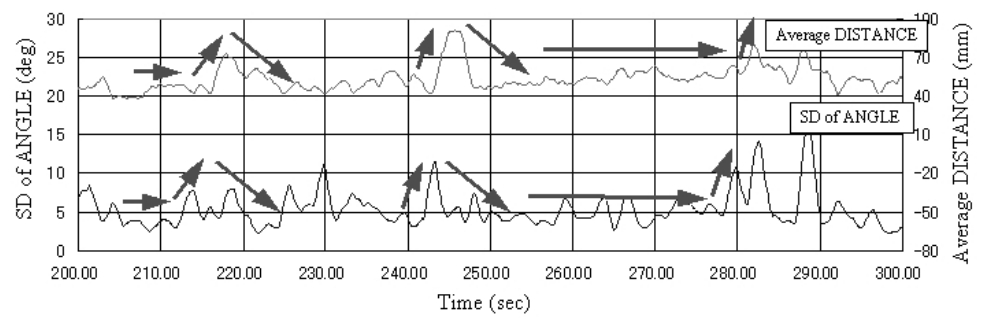

Fig. 3. Moving average of DISTANCE and standard deviation of ANGLE

\subsection{Results and Discussion}

This experiment involved four expert surgeons (A, B, C and D). The results of the DISTANCE-ANGLE distributions are presented in the form of histograms (Figure 2). There was little difference between surgeon-assistant pairs A-B and C-D for both DISTANCE and ANGLE, a result that suggests the criteria for maneuvering the laparoscope may be human-independent (i.e., doctor-independent). Then, we investigated the correlation between DISTANCE and ANGLE. Figure 3 shows a portion of the time sequence of the moving average of DISTANCE and the moving standard deviation of ANGLE for surgeon-assistant pair C-D. We chose the moving window size to be 20 data (corresponding to 1 second at the tracking rate of $20 \mathrm{~Hz}$ ). It can be seen that the average of DISTANCE and the standard deviation (SD) of ANGLE have a high correlation. The SD of ANGLE at first increases/decreases rapidly, then the average of DISTANCE also increases/decreases, and consequently the SD of ANGLE becomes stable. The SD of ANGLE corresponds to the 2D distribution of the projection of the right-hand instrument onto the laparoscopic image, so it can be estimated in 


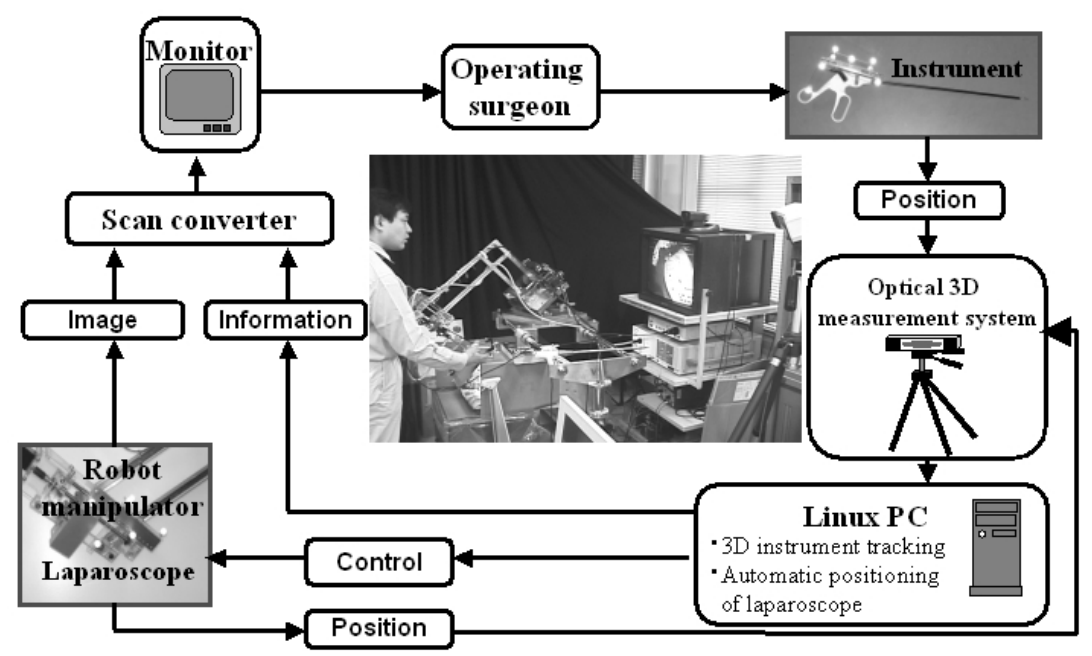

Fig. 4. Robotic laparoscope positioning system

real time by monitoring laparoscopic images. The camera assistant can easily control the SD of ANGLE by inserting/retracting the laparoscope, which causes the decrease/increase of DISTANCE. Thus, we hypothesized that the camera assistant always observed the SD of ANGLE and controlled the zooming ratio of laparoscopic images such that the SD of ANGLE would stay within an appropriate range.

\section{Implementation of Zooming Behavior of Camera Assistants}

\subsection{Robot System Overview}

We implemented our abovementioned hypothesis on the robotic laparoscope positioner we previously developed $[\underline{6}$. The system configuration is shown in Figure 4. Our laparoscope positioning system consists primarily of an optical 3D measurement system (Polaris Accedo, NDI Corporation), an all-purpose PC (CPU: Intel R Core 2 Duo E6600 2.4GHz, Memory: 2GB, OS: Vine Linux 4.0), a robot manipulator with three degrees-of-freedom that holds the laparoscope (for details, see [8), a scan converter (DSC06d-HR, Digital Arts Corporation) for superimposing graphics on the scope image, and a 21-inch TV monitor.

Initially in this system, DISTANCE is set as the base distance $d_{0}$, and the position of the laparoscope is controlled by the following algorithm (referred to as "Proposed") under the condition that DISTANCE is kept within $\left[d_{\min }, d_{\max }\right]$.

- The system starts centering the tip of the right-hand instrument in the image when ANGLE is more than $\theta_{\max }$, and stops it when ANGLE is less than a threshold $\theta_{t h}$. 


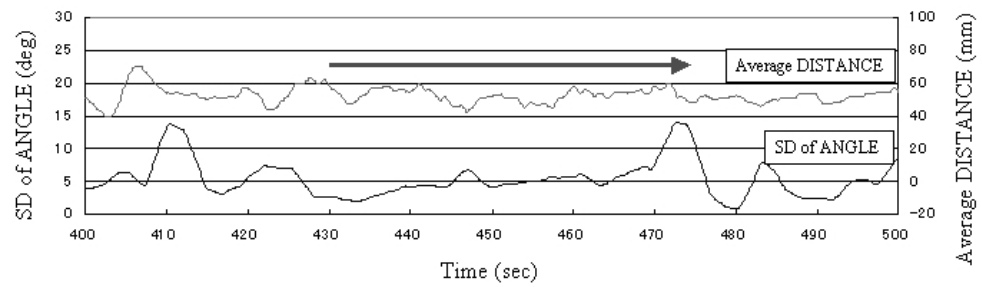

Fig. 5. Moving average of DISTANCE and SD of ANGLE (Conventional)

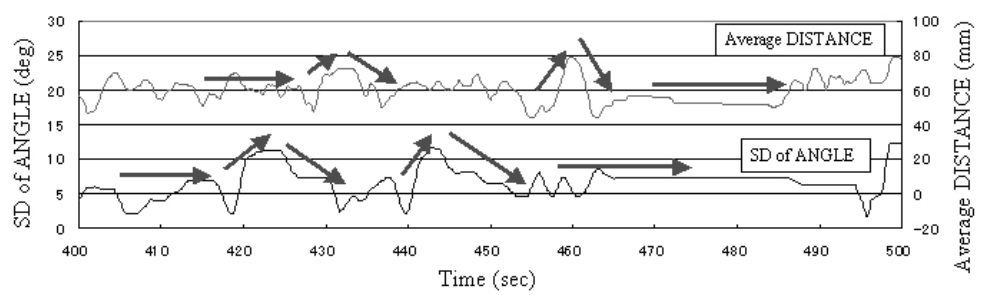

Fig. 6. Moving average of DISTANCE and SD of ANGLE (Proposed)

- Also, the system starts inserting/retracting the laparoscope when the SD of ANGLE is without the range $R:\left[\sigma_{\min }, \sigma_{\max }\right]$, and stops it when the SD of ANGLE is within the range $R$.

Based on the data of human camera assistants, as shown in Figures 2 and 3 , we set $d_{0}=50 \mathrm{~mm}, d_{\min }=30 \mathrm{~mm}, d_{\max }=70 \mathrm{~mm}, \theta_{\max }=20 \mathrm{deg}, \sigma_{\min }=3 \mathrm{deg}$, $\sigma_{\max }=5.5 \mathrm{deg}$. Also, we set the threshold for image centering $\theta_{t h}=2 \mathrm{deg}$.

For comparison, we also implemented a "uniform" distance algorithm (referred to as "Conventional"). This algorithm inserts/retracts the laparoscope such that DISTANCE is always the base distance $d_{0}$ while centering the tip of the righthand instrument in the image.

\subsection{Results and Discussion}

To evaluate the validity of the proposed system, a laparoscopic cholecystectomy simulation with a pig organ was performed by surgeons $\mathrm{C}$ and $\mathrm{B}$. In addition, the same experiment was conducted using the conventional algorithm. In all experiments, the surgeon successfully and safely completed the entire operative procedure without the support of the human camera assistant. As shown in Figure 5, the average of DISTANCE and SD of ANGLE had no correlation in the case of using the conventional algorithm. In contrast, the zooming behavior of the proposed robot system (Figure 6) was very similar to that of the human camera assistant (Figure 33).

Figure 7 illustrates the comparison between the conventional method and the proposed one using three kinematic parameters: (a) Time, (b) Path length, and 


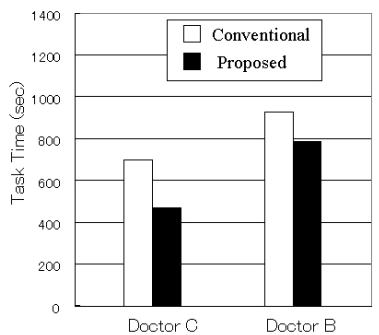

(a) Time

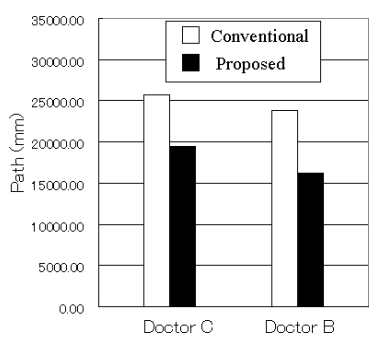

(b) Path length

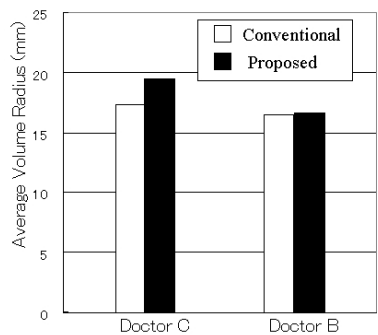

(c) Average volume radius

Fig. 7. Comparison between the conventional and the proposed methods

(c) Average volume radius. "Time" indicates the total time required to perform the task and "Path length" indicates the length of the trajectory outlined by the tip of the instrument over time. This parameter describes the spatial distribution of the tip of the laparoscopic instrument in the workspace of the task 9. "Average volume radius" is a calculation of the average distance between the three-dimensional position of the tip of the instrument and the centroid of the trajectory described by the tip of the instrument over time. This parameter corresponds to the size of the workspace of the task.

For both subjects, both "time" and "path length" were shorter for the proposed method compared with those for the conventional method, whereas there was little difference in the "average volume radius" between the methods. These results suggest that the proposed zooming motion may be suitable for surgical actions such as fast and compact operations.

\section{Conclusion}

In this paper, it was found that the $2 \mathrm{D}$ distribution of the projection of the right-hand surgical instrument onto the laparoscopic image (called the SD of ANGLE) is one of the key factors in which camera assistants determine the zooming ratio of laparoscopic images during surgery. We are now investigating another key factor for deciding the zooming ratio by analyzing the positional relationship between the laparoscope and the "left-hand" surgical instrument as well as the "right-hand" one. We are planning to implement the proposed method in another robotic laparoscope positioner [10] with the goal of in vivo and clinical applications.

\section{Acknowledgements}

This research was supported in part by "Special Coordination Funds for Promoting Science and Technology: Yuragi Project" of the Ministry of Education, Culture, Sports, Science and Technology, Japan, and Grant-in-Aid for Scientific Research (A) (No.19206047) of the Japan Society for the Promotion of Science. 


\section{References}

1. Wei, G.Q., Arbter, K., Hirzinger, G.: Real-time visual servoing for laparoscopic surgery. IEEE Engineering in Medicine and Biology Magazine 16, 40-45 (1997)

2. Casals, A., Amat, J., Laporte, E.: Automatic guidance of an assistant robot in laparoscopic surgery. In: Proceedings of the 1996 IEEE International Conference on Robotics and Automation, pp. 895-900 (1996)

3. Wang, Y.F., Uecker, D.R., Wang, Y.: A new framework for vision-enabled and robotically assisted minimally invasive surgery. Computerized Medical Imaging and Graphics 22, 429-437 (1998)

4. Nishikawa, A., Asano, S., Fujita, R., Yamaguchi, S., Yohda, T., Miyazaki, F., Sekimoto, M., Yasui, M., Takiguchi, S., Miyake, Y., Monden, M.: Selective use of face gesture interface and instrument tracking system for control of a robotic laparoscope positioner. In: Ellis, R.E., Peters, T.M. (eds.) MICCAI 2003. LNCS, vol. 2879, pp. 973-974. Springer, Heidelberg (2003)

5. Ko, S.Y., Kwon, D.S.: A surgical knowledge based interaction method for a laparoscopic assistant robot. In: Proceedings of the 13th IEEE International Workshop on Robot and Human Interactive Communication (Ro-Man 2004), pp. 313-318 (2004)

6. Nishikawa, A., Ito, K., Nakagoe, H., Taniguchi, K., Sekimoto, M., Takiguchi, S., Seki, Y., Yasui, M., Okada, K., Monden, M., Miyazaki, F.: Automatic positioning of a laparoscope by preoperative workspace planning and intraoperative 3D instrument tracking. In: MICCAI 2006 Workshop Proceedings, Workshop on Medical Robotics: Systems and Technology towards Open Architecture, pp. 82-91 (2006)

7. Blasinski, H., Nishikawa, A., Miyazaki, F.: The application of adaptive filters for motion prediction in visually tracked laparoscopic surgery. In: Proceedings of 2007 IEEE International Conference on Robotics and Biomimetics (ROBIO 2007), pp. 360-365 (2007)

8. Nishikawa, A., Hosoi, T., Koara, K., Negoro, D., Hikita, A., Asano, S., Kakutani, H., Miyazaki, F., Sekimoto, M., Yasui, M., Miyake, Y., Takiguchi, S., Monden, M.: FAce MOUSe: A novel human-machine interface for controlling the position of a laparoscope. IEEE Trans. on Robotics and Automation 19(5), 825-841 (2003)

9. Cortin, S., Stylopoulos, N., Ottensmeyer, M., Neumann, P., Rattner, D., Dawson, S.: Metrics for laparoscopic skill trainers: The weakest link! In: Dohi, T., Kikinis, R. (eds.) MICCAI 2002. LNCS, vol. 2488, pp. 35-43. Springer, Heidelberg (2002)

10. Taniguchi, K., Nishikawa, A., Miyazaki, F., Kobayashi, T., Kazuhara, K., Ichihara, T., Sekimoto, M., Takiguchi, S., Monden, M.: Development of a safe disposable laparoscope manipulator using hydraulic actuators. In: Proceedings of 2007 IEEE International Conference on Robotics and Biomimetics (ROBIO 2007), pp. 601-606 (2007) 